\title{
Quality of life and other patient-reported outcomes in adult Lebanese patients with type 2 diabetes during COVID-19 pandemic
}

\author{
E. Naous ${ }^{1}$ (1) $\cdot$ M. Boulos ${ }^{1} \cdot$ G. Sleilaty ${ }^{2} \cdot$ A. A. Achkar ${ }^{1} \cdot$ M.-H. Gannagé-Yared ${ }^{1}$
}

Received: 20 September 2021 / Accepted: 1 November 2021 / Published online: 15 November 2021

(C) Italian Society of Endocrinology (SIE) 2021

\begin{abstract}
Introduction Several studies have shown that COVID-19 pandemic has a negative impact on type 2 diabetic mellitus (T2DM) patients' quality of life (QoL). However, very few studies were performed in Middle Eastern countries.

Aim The aim of the current study was to assess, the QoL and diabetes-specific QoL, treatment satisfaction and psychological distress of Lebanese patients with T2DMs using: the Audit of Diabetes-Dependent Quality of Life (ADDQoL), Diabetes Treatment Satisfaction Questionnaire status version (DTSQs) and Kessler 10 (K10) questionnaires and to compare results to those obtained during the pre-COVID-19 period.

Results 461 patients with T2DM participated in the study; $52.6 \%$ men, $47.4 \%$ women; median age 59 years old. The respective median ADDQoL and DTSQs scores were - 2.2 (interval interquartile range (IQR) - 3.9, - 0.8) (range from - 9 maximum negative impact to +3 maximum positive impact) and 30(IQR22-36) (range from 0 maximum dissatisfaction to 36 maximum satisfaction). K10 median score was 26(IQR18-35) (range from minimum score of 10 indicating no distress to maximum score of 50 indicating severe distress). Rural dwelling, lack of exercise, current smoking, diabetic complications, injectable diabetes treatment, and previous COVID-19 infection were all associated with significantly worse ADDQoL, DTSQs, and K10 score indicating greater distress. A significant worsening of ADDQoL scores followed onset of the pandemic with no significant change in DTSQs scores.

Conclusion During the COVID-19 pandemic, T2DM Lebanese patients experienced more negative impact of diabetes on QoL and mental health. Those infected with COVID-19 also reported worse QoL, treatment satisfaction and mental health. This highlights the need for community and individual support.
\end{abstract}

Keywords Type 2 diabetes · Quality of life · Treatment satisfaction $\cdot$ Mental health $\cdot$ Lebanon

\section{Introduction}

In November 2019, the novel coronavirus disease 2019 (COVID-19) outbreak started in the Hubei Province in China. Since then and within a very short lapse of time, the virus has spread to multiple countries across the globe [1].

E. Naous and M. Boulos contributed equally to this work and should be considered co-first author.

E. Naous

elie.naous@net.usj.edu.lb

1 Department of Endocrinology, Saint-Joseph University, Faculty of Medicine, Beirut, Lebanon

2 Department of Biostatistics and Clinical Research Center, Faculty of Medicine, Saint-Joseph University, Beirut, Lebanon
On the 11th of March 2020, the World Health Organization declared the spread of COVID-19 a global pandemic [1]. The manifestations of COVID-19 vary between mild symptoms such as a common cold to severe respiratory infections and multiple organ failure [2]. The presence of chronic diseases such as obesity, hypertension, and/or diabetes mellitus are considered as high-risk factors for increased severity and mortality from the infection [3]. It was estimated that $10 \%$ of patients infected with COVID-19 have type 2 diabetes mellitus (T2DM) and that there was a two-fold increase in the rate of intensive care unit admission in individuals with T2DM compared to those without diabetes [4]. The pandemic has severely disrupted routine diabetes self-management because of changes in diet due to unavailability of certain food products and difficulty in maintaining physical exercise due to the lockdown. Furthermore, people with 
T2DM were facing difficulties visiting doctors and refilling prescriptions [5].

It is well known that T2DM causes a serious deterioration in general Quality of Life (QoL) by affecting mental, physical, and social health QoL components [6-8]. Several studies have shown that COVID-19 pandemic has an additional negative impact on the QoL and treatment satisfaction of people with T2DM [9-13]. Moreover, depression, anxiety, and panic attacks are more commonly seen in people with T2DM during COVID-19 [10, 13-15].

Data related to the QoL in T2DM from Middle Eastern countries during the COVID-19 pandemic are scarce [11, 13, 16]. Only three studies from Egypt [11], Iran [13], and Saudi Arabia [16] were performed during the COVID-19 pandemic and showed an altered QoL in T2DM patients. In Lebanon, a single study performed in 2017 [17] reported the QoL and treatment satisfaction in Lebanese and Jordanian adults with T2DM. The authors found using the Audit of Diabetes-Dependent Quality of life (ADDQoL), the Diabetes Treatment Satisfaction Questionnaire status version (DTSQs), and the Hypoglycemia Fear Survey-II (HFS-II) questionnaire a greater negative impact on QoL, dissatisfaction with treatment and fear of hypoglycemia in patients with T2DM.

This study aims to evaluate, for the first time, the QoL and the impact of diabetes on QoL, treatment satisfaction and mental health status, in a sample of the Lebanese T2DM population during the Covid-19 pandemic. In addition, the study will compare our results to those of the 2017 report.

\section{Materials and methods}

\section{Participants}

Three questionnaires were distributed to T2DM patients in the waiting rooms when consulting their physicians in hospitals and private clinics across the Lebanese territory, between January and June 2021. Sociodemographic data were collected from each participant. Information included age, gender, marital and educational status, dwelling location, type of medical insurance coverage, and income. The medical history was recorded from medical charts: presence of hypertension or dyslipidemia, smoking habits, physical activity, previous infection with COVID-19, and diabetesrelated information: duration of the disease, current diabetes treatment (oral, injectable or both), last glycated hemoglobin (HBA1c), and presence of diabetes-related complications: nephropathy, retinopathy, and neuropathy. For each patient, weight and height were also recorded from medical charts. Body Mass Index (BMI) was calculated as the weight in kilograms divided by the square of height in meters.

\section{Questionnaires}

Three questionnaires were used for the study: the ADDQoL and the DTSQs linguistically validated in Arabic for Lebanon, and the Kessler Psychological Distress Scale (K10) in a validated Arabic version.

The ADDQoL questionnaire $[18,19]$ evaluates QoL and the impact of diabetes on the QoL. It is composed of two overview items, the first (overview item I) measures the general QoL and the second (overview item II) measures the overall impact of diabetes on the individuals' QoL. The overview items are followed by a series of 19 questions about the impact of diabetes on leisure, work, journeys, physical health, family life, friendships and social life, personal relationship, sexuality, physical appearance, selfconfidence, motivation, reactions of other people, feelings about the future, finances, living conditions, dependence on other people, dietary freedom. This evaluation allows patients to indicate which aspects of life apply to them, the impact of diabetes on each applicable aspect of life (including any positive as well as negative impact on a scale from -3 maximum negative impact to +1 positive impact); and the perceived importance of each aspect of life for their QoL (from 3 very important to 0 not at all important). Impact scores are multiplied by importance scores to give weighted impact scores for all applicable items ranging from -9 to +3 . An Average Weighted Impact (AWI) score can be obtained by summing the weighted impact scores for all applicable items and dividing by the number of applicable items for the individual.

The DTSQs $[20,21]$ is a globally accepted and used tool [22], recommended by the World Health Organization and the International Diabetes Federation [23], to evaluate treatment satisfaction in T2DM patients. It was designed to assess treatment satisfaction in specific areas, treatment satisfaction from these areas combined and perceived frequency of hyperglycemia and hypoglycemia. The six questions which are combined to assess treatment satisfaction ask about "satisfaction with current treatment", "flexibility", "convenience", "understanding of diabetes", "recommend treatment to others" and "willingness to continue". Each item is rated on a 7-point scale from 6 (e.g., "very satisfied") to 0 (e.g., "very dissatisfied"). Treatment satisfaction is assessed as the sum of the scores of these six questions (maximum score 36 , minimum score 0 ), with a higher score indicating greater treatment satisfaction. Two additional items, scored as separate items, assess the frequency of hyper- and hypoglycemia (Questions 2 and 3 ). The scale of answers ranges from 6 "most of the time" to 0 "none of the time".

Finally, the K10 scale [24] was used to provide a measure of mental distress. It consists of a 10-item 
questionnaire evaluating anxiety and depressive symptoms experienced during the last 4 -week period. The score varies from 10 to 50. Respective scores lower than 20, indicates good mental health while 20 to 24 indicates a mild disorder, 25 to 29 a moderate and more than 30 indicates a severe mental disorder.

\section{Statistical analyses}

The distribution of the continuous variables was checked using the Shapiro-Wilk (SW) test, with additional inspection of quartile-quartile plots. Native variables with a skewed distribution were expressed as median with its interval interquartile range (IQR) (1st quartile (Q1)-3rd quartile (Q3) while normally distributed variables were expressed as mean \pm standard deviation (SD). For the analysis of the ADDQoL, the AWI score was used. The correlation between the 3 scores (AWI ADDQoL, DTSQs Total score and K10) was estimated using the Spearman correlation coefficient. The three scores were evaluated between the different categories of demographic and anthropometric factors, lifestyle, and medical history, as well as according to the presence of distinct diabetic complications, using the Mann-Whitney U test and the Kruskall-Wallis test as appropriate. The 3 scores were also correlated with age, educational level, income, BMI, duration of diabetes and last HbA1c level, using Spearman correlation coefficient. The comparison with the 2017 report [17] relied on the independent samples T test.

The statistical analysis was performed using IBM SPSS (IBMCorp; SPSS Statistics for Windows v26.1, 2019, Armonk, NY, USA).

\section{Ethical considerations}

The study was approved by the Ethics Committee of SaintJoseph University (Tfem 2022/11) and all the volunteers signed a written informed consent form before completing the questionnaire.

\section{Results}

\section{Demographic, anthropometric, and clinical characteristics of the participants}

461 Lebanese patients with T2DM were included in the study (52.6\% men, $47.4 \%$ women). Table 1 summarizes the patients' characteristics. The median age was 59 (IQR 48-65) years, and the median BMI 28.1 (IQR 25.3-32.0) kg/ $\mathrm{m}^{2}$. Hypertension was found in $52.3 \%$ of the sample, $49.9 \%$ had dyslipidemia and $41.0 \%$ were current smokers. Most of the participants (65\%) reported no physical activity. The median diabetes duration was 10 (IQR 3.0-16.0) years and
Table 1 Patient characteristics: demographic, anthropometric and clinical

\begin{tabular}{|c|c|}
\hline Age, year $(Q 1-Q 3)$ & $59(48-65)$ \\
\hline Body mass index, $\mathrm{kg} / \mathrm{m}^{2}(Q 1-Q 3)$ & $28.1(25.3-32.0)$ \\
\hline \multicolumn{2}{|l|}{ Gender } \\
\hline Men, $n(\%)$ & $241(52.6)$ \\
\hline Women, $n(\%)$ & $217(47.4)$ \\
\hline \multicolumn{2}{|l|}{ Education level } \\
\hline Primary school, $n(\%)$ & $121(26.6)$ \\
\hline Secondary school, $n(\%)$ & $139(30.5)$ \\
\hline University, $n(\%)$ & $195(42.9)$ \\
\hline \multicolumn{2}{|l|}{ Income } \\
\hline None, $n(\%)$ & $78(17.1)$ \\
\hline Insufficient, $n(\%)$ & $143(31.4)$ \\
\hline Sufficient, $n(\%)$ & $214(47.0)$ \\
\hline More than sufficient, $n(\%)$ & $20(4.4)$ \\
\hline \multicolumn{2}{|l|}{ Type of medical coverage } \\
\hline None, $n(\%)$ & $18(4.4)$ \\
\hline Private insurance, $n(\%)$ & $110(24.7)$ \\
\hline Public, $n(\%)$ & $319(70.9)$ \\
\hline \multicolumn{2}{|l|}{ Dwelling location } \\
\hline Beirut, $n(\%)$ & $102(22.4)$ \\
\hline Mount Lebanon, $n(\%)$ & $193(42.3)$ \\
\hline South, $n(\%)$ & $23(5.0)$ \\
\hline North, $n(\%)$ & $129(28.3)$ \\
\hline Bekaa, $n(\%)$ & $9(2.0)$ \\
\hline \multicolumn{2}{|l|}{ Marital status } \\
\hline Married, $n(\%)$ & $269(60.6)$ \\
\hline Single, $n(\%)$ & $105(23.6)$ \\
\hline Others, $n(\%)$ & $70(36.0)$ \\
\hline Presence of hypertension, $n(\%)$ & $239(52.3)$ \\
\hline Presence of dyslipidemia, $n(\%)$ & $228(49.9)$ \\
\hline Current smoking, $n(\%)$ & $188(41.0)$ \\
\hline \multicolumn{2}{|l|}{ Physical activity } \\
\hline None, $n(\%)$ & $299(65.1)$ \\
\hline Less than $1 /$ week, $n(\%)$ & $25(5.4)$ \\
\hline 1/week, $n(\%)$ & $60(13.1)$ \\
\hline More than $1 /$ week, $n(\%)$ & $43(9.4)$ \\
\hline Daily, $n(\%)$ & $32(7.0)$ \\
\hline Previous COVID-19 infection, $n(\%)$ & $172(37.4)$ \\
\hline
\end{tabular}

Categorical variables are presented as frequency and its percentage. Continuous variables whose distribution deviates from normality and ordinal variables are presented as the median with its interquartile range $(Q 1-Q 3)$

the last median tested HbA1c 7.0\% (IQR 6.0-8.0). Most of the patients were treated with oral anti-diabetes drugs (OAD) exclusively (77.7\%), 15.7\% were using injectable therapy alone, and $6.4 \%$ were using combined oral and injectable therapy. The most common observed diabetic complication was neuropathy $(14.3 \%)$ followed by nephropathy $(11.7 \%)$ and retinopathy $(6.3 \%)$. 


\section{Quality of life, treatment satisfaction, and mental health}

Results of the ADDQoL, DTSQs, and K10 scores are shown in Table 2. A higher ADDQoL and DTSQs scores and a lower K10 score indicate better QoL, treatment satisfaction and mental health. Out of 461 patients, 7 (1.5\%) did not complete the ADDQoL questionnaire, 19 (3.9\%) did not complete the DTSQs, and $5(1.1 \%)$ did not complete the K10 questionnaire. The range of AWI score given on the ADDQoL questionnaire varied from -9 to +0.2 with a median of -2.2 (IQR $-3.9,-0.8$ ). $53.3 \%$ of the patients describe their general QoL (overview item I) as less than good and $80.5 \%$ estimate that if they did not have diabetes, their QoL would improve (overview item II).

For the DTSQs, the median score was 30 (IQR 22-36). For both, the perceived frequency of hyper- and hypoglycemia items, the median score was 3 (IQR 1-5).

The median score of the K10 questionnaire was 26 (IQR 18-35).

The ADDQoL score was positively correlated with the DTSQs score $(r=0.30, p<0.001)$ and inversely correlated with the K10 score $(r=-0.32, p<0.001)$. Furthermore, the
DTSQs score was inversely correlated with the K10 score $(r=-0.46, p<0.001)$. Finally, the scores of perceived hyperand hypoglycemia were inversely correlated with ADDQoL (respectively $r=-0.22, p<0.001$ and $r=-0.14 . p=0.003$ ) and positively correlated with the K10 score (respectively $r=0.25, p<0.001$ and $r=0.21, p<0.001$ ). No significant correlation was found between the score of perceived hyperor hypoglycemia and the DTSQs treatment satisfaction score.

\section{Factors influencing patient's QoL, treatment satisfaction and mental health status}

Table 3 shows the patients' scores for the ADDQoL, DTSQs, and K10 questionnaires according to sociodemographic and clinical characteristics.

\section{Effect of sociodemographic and anthropometric parameters}

No gender difference was observed for the ADDQoL and DTSQs scores. However, the K10 score was significantly higher in women compared to men $(p=0.007)$. Age was
Table 2 ADDQol, DTSQs, and K10 patient's scores

\begin{tabular}{|c|c|c|}
\hline & $n(\%)$ & Median $(Q 1-Q 3)$ \\
\hline ADDQoL average weighted impact score total & & $-2.2(-3.9,-0.8)$ \\
\hline Overview item I: in general, my present quality of life is & $457(100)$ & $0(0-1)$ \\
\hline Extremely bad, $n(\%)$ & $8(1.8)$ & \\
\hline Very bad, $n(\%)$ & $19(4.2)$ & \\
\hline Bad, $n(\%)$ & $47(10.3)$ & \\
\hline Neither good nor bad, $n(\%)$ & $169(37.0)$ & \\
\hline Good, $n(\%)$ & $145(31.7)$ & \\
\hline Very good, $n(\%)$ & $47(10.3)$ & \\
\hline Excellent, $n(\%)$ & $22(4.8)$ & \\
\hline Overview item II: if I did not have diabetes, my quality of life would be & $451(100)$ & $-1(-2,-1)$ \\
\hline Very much better, $n(\%)$ & $56(12.4)$ & \\
\hline Much better, $n(\%)$ & $146(32.4)$ & \\
\hline A little better, $n(\%)$ & $161(35.7)$ & \\
\hline The same, $n(\%)$ & $80(17.7)$ & \\
\hline Worse, $n(\%)$ & $8(1.8)$ & \\
\hline DTSQs total (for the 6 questions: questions $1,4,5,6,7$ and 8 ) & $443(100)$ & $30(22-36)$ \\
\hline Perceived frequency of hyperglycemia (question 2) & $434(100)$ & $3(1-5)$ \\
\hline Perceived frequency of hypoglycemia (question 3) & $434(100)$ & $3(1-5)$ \\
\hline K10 score & $456(100)$ & $26(18-35)$ \\
\hline$<20$ : well mental health & $125(27.4)$ & \\
\hline 20 to 24 : mild mental disorder & $87(19.1)$ & \\
\hline 25 to 29: moderate mental disorder & $69(15.1)$ & \\
\hline$>30$ : severe mental disorder & $175(38.4)$ & \\
\hline
\end{tabular}

Categorical variables are presented as frequency and its percentage

Continuous variables which distribution deviates from normality and ordinal variables are presented as median with its interquartile range $(Q 1-Q 3)$ 
Table 3 ADDQoL, DTSQs, and K10 scores according to sociodemographic and clinical parameters

\begin{tabular}{|c|c|c|c|c|c|c|c|}
\hline & & \multicolumn{2}{|c|}{$\begin{array}{l}\text { ADDQoL: average weighted } \\
\text { impact }\end{array}$} & \multicolumn{2}{|c|}{$\begin{array}{l}\text { DTSQs scale score }(1,4,5 \text {, } \\
6,7,8)\end{array}$} & \multicolumn{2}{|l|}{ K10 total } \\
\hline & & Median $(Q 1-Q 3)$ & $p$ value & Median $(Q 1-Q 3)$ & $p$ value & Median $(Q 1-Q 3)$ & $p$ value \\
\hline \multirow[t]{2}{*}{ Gender } & Men & $-2.2(-4.0,-0.6)$ & 0.46 & $30(23-36)$ & 0.26 & $25(17-33)$ & 0.007 \\
\hline & Women & $-2.19(-3.9,-1.0)$ & & $29(21-36)$ & & $27(20-36)$ & \\
\hline \multirow[t]{3}{*}{ Education } & Primary & $-1.9(-3.6,-1.1)$ & 0.29 & $30(22-36)$ & 0.74 & $28(22-36)$ & $<0.001$ \\
\hline & Secondary & $-2.6(-4.0,-1.0)$ & & $29(23-36)$ & & $27(17-37)$ & \\
\hline & University & $-1.9(-4.0,-0.5)$ & & $30(21-36)$ & & $24(16-31)$ & \\
\hline \multirow[t]{4}{*}{ Income } & None & $-2.1(-3.8,-0.9)$ & 0.16 & $26(19-33)$ & 0.001 & $30(22-40)$ & $<0.001$ \\
\hline & Insufficient & $-2.6(-4.0,-1.1)$ & & $28(21-36)$ & & $29(22-40)$ & \\
\hline & Sufficient & $-1.8(-3.8,-0.6)$ & & $32(24-36)$ & & $24(16-31)$ & \\
\hline & More than sufficient & $-2.9(-5.2,-1.2)$ & & $29(22-36)$ & & $17(12-27)$ & \\
\hline \multirow[t]{3}{*}{ Coverage } & None & $-3.4(-3.9,-1.7)$ & 0.21 & $31(23-35)$ & 0.36 & $34(23-36)$ & 0.38 \\
\hline & Private insurance & $-1.8(-4.9,-1.0)$ & & $31(23-36)$ & & $27(20-35)$ & \\
\hline & Public & $-2.3(-3.9,-1.7)$ & & $29(21-36)$ & & $25(18-35)$ & \\
\hline \multirow[t]{5}{*}{ Dwelling location } & Beirut & $-2.6(-3.9,-1.0)$ & $<0.001$ & $31(24-36)$ & $<0.001$ & $26(22-34)$ & $<0.001$ \\
\hline & Mount Lebanon & $-1.7(-3.1,-0.6)$ & & $33(26-36)$ & & $21(15-28)$ & \\
\hline & South & $-2.7(-4.2,-1.3)$ & & $32(24-36)$ & & $33(20-41)$ & \\
\hline & North & $-3.1(-5.7,-0.9)$ & & $24(16-32)$ & & $30(24-40)$ & \\
\hline & Bekaa & $-2.6(-4.1,-2.3)$ & & $31(23-36)$ & & $35(27-40)$ & \\
\hline \multirow[t]{2}{*}{ Marital status } & Married & $-2.3(-3.9,-0.7)$ & 0.64 & $28(21-36)$ & $<0.016$ & $24(17-34)$ & 0.34 \\
\hline & Single & $-2.0(-4.0,-0.8)$ & & $33(25-36)$ & & $27(20-33)$ & \\
\hline \multirow[t]{2}{*}{ Hypertension } & Yes & $-2.4(-4.1,-0.8)$ & 0.16 & $26(20-34)$ & $<0.001$ & $25(17-34)$ & 0.77 \\
\hline & No & $-1.9(-3.9,-0.8)$ & & $32(24-36)$ & & $26(20-33)$ & \\
\hline \multirow[t]{2}{*}{ Dyslipidemia } & Yes & $-2.6(-4.3,-1.0)$ & 0.013 & $27(20-35)$ & $<0.001$ & $26(20-37)$ & 0.034 \\
\hline & No & $-1.8(-3.8,-0.7)$ & & $32(24-36)$ & & $25(18-33)$ & \\
\hline \multirow[t]{2}{*}{ Current smoking } & Yes & $-2.7(-4.5,-1.1)$ & 0.001 & $26(19-33)$ & $<0.001$ & $30(20-36)$ & $<0.001$ \\
\hline & No & $-1.8(-3.5,-0.7)$ & & $33(24-36)$ & & $24(17-31)$ & \\
\hline \multirow[t]{5}{*}{ Physical activity } & None & $-2.2(-3.8,-1.0)$ & 0.031 & $28(20-36)$ & 0.091 & $28(20-36)$ & 0.006 \\
\hline & $<$ Once per week & $-3.9(-5.3,-1.1)$ & & $27(23-34)$ & & $25(22-36)$ & \\
\hline & Once per week & $-2.3(-3.9,-0.6)$ & & $33(24-36)$ & & $25(17-32)$ & \\
\hline & $>$ Once per week & $-1.0(-3.5,-0.2)$ & & $34(29-36)$ & & $18(12-26)$ & \\
\hline & Daily & $-2.0(-4.4,-0.5)$ & & $33(23-36)$ & & $20(10-25)$ & \\
\hline \multirow[t]{3}{*}{ Diabetes Treatment } & Oral & $-1.9(-3.9,-0.8)$ & 0.030 & $31(22-36)$ & 0.001 & $25(18-34)$ & 0.011 \\
\hline & Injectable & $-2.8(-4.1,-1.5)$ & & $26(17-33)$ & & $30(23-37)$ & \\
\hline & Oral+ injectable & $-3.0(-4.3,-1.4)$ & & $26(23-32)$ & & $28(19-38)$ & \\
\hline \multirow[t]{2}{*}{ Nephropathy } & Yes & $-4.0(-5.8,-2.4)$ & $<0.001$ & $20(13-24)$ & $<0.001$ & $40(30-43)$ & $<0.001$ \\
\hline & No & $-1.9(-3.7,-0.8)$ & & $31(24-36)$ & & $24(17-33)$ & \\
\hline \multirow[t]{2}{*}{ Retinopathy } & Yes & $-3.9(-6.2,-2.8)$ & $<0.001$ & $16(6-25)$ & $<0.001$ & $36(30-44)$ & $<0.001$ \\
\hline & No & $-2.0(-3.8,-0.8)$ & & $30(23-36)$ & & $25(18-34)$ & \\
\hline \multirow[t]{2}{*}{ Neuropathy } & Yes & $-3.3(-5.7,-2.3)$ & $<0.001$ & $23(12-30)$ & $<0.001$ & $36(27-43)$ & $<0.001$ \\
\hline & No & $-1.8(-3.8,-0.8)$ & & $30(23-36)$ & & $24(17-33)$ & \\
\hline \multirow[t]{2}{*}{ Previous COVID-19 infection } & Yes & $-2.6(-4.9,-0.9)$ & 0.027 & $27(18-35)$ & 0.001 & $28(21-37)$ & 0.001 \\
\hline & No & $-2.0(-3.6,-0.8)$ & & $31(24-36)$ & & $24(17-32)$ & \\
\hline
\end{tabular}

only significantly correlated with the ADDQoL score indicating better QoL with age $(r=0.12, p=0.012)$. BMI was not significantly correlated with the three questionnaire scores. Living outside the city of Beirut was associated with lower scores in ADDQoL and DTSQs, and with 
higher score in K10 questionnaire $(p<0.001$ for the three scores).

\section{Effect of lifestyle, health insurance coverage, and diabetes characteristics}

No significant differences in DTSQs scores were observed in patients with no physical activity compared to those who exercise regularly. However, lower ADDQoL and K10 scores were noted in patients with low physical activity (respectively $p=0.003$ and $=0.003$ ). Current smoking was associated with significant lower scores in ADDQoL and DTSQs, and higher score in the K10 questionnaire (respectively $p=0.001, p<0.001$ and $p<0.001)$ indicating more negative impact of diabetes on QoL and treatment satisfaction and worse mental health in smokers. No significant differences in ADDQoL, DTSQ and K10 scores were observed according to medical insurance coverage. The level of last measured $\mathrm{HbA} 1 \mathrm{c}$ was positively correlated with the K10 score $(r=0.21, p<0.001)$ and inversely correlated with ADDQoL $(r=-0.26, p<0.001)$, and DTSQs $(r=-0.43$, $p<0.001)$ scores, indicating that lower $\mathrm{HbA} 1 \mathrm{c}$ is associated with better mental health, reduced impact of diabetes on QoL and better treatment satisfaction. Diabetes duration was not significantly correlated with the three questionnaires' scores. Treatment with OAD exclusively, the absence of dyslipidemia, and diabetes complications (nephropathy, retinopathy, and neuropathy) were all significantly associated with higher scores in ADDQoL and DTSQs, and lower score in K10 questionnaires. Finally, previous COVID-19 infection was associated with lower ADDQoL and DTSQs scores, and higher K10 score (respective p values 0.027, 0.001, 0.001).

\section{ADDQoL and DTSQs scores during COVID-19 versus pre-COVID-19}

The comparison between the results of the current study and those of Atallah et al. [17] are shown in Table 4. A significant difference was seen in ADDQoL AWI score, overview
I, and II items ( $p=0.001, p<0.001$, and $p<0.001$ respectively). $53 \%$ of our population (versus $25 \%$ in the Atallah et al. study) described their general QoL as below good in the ADDQoL overview I item $(p<0.001)$. For the overview II item, $80 \%$ of our population (vs $90 \%$ in Atallah et al. study) thought that if they did not have diabetes, their QoL would improve to a different extent $(p<0.001)$. No difference was noted between the two DTSQs scores $(p=0.61)$. In addition, only $35 \%$ of our patients practice regular physical activity versus $44 \%$ in the Atallah et al. study $(p=0.002)$. An increase in smoking habits was also noted in our population during the lockdown since $27.7 \%$ of the Atallah et al. were current smokers versus $41 \%$ in our population $(p<0.001)$.

\section{Discussion}

Our results have shown that the QoL, treatment satisfaction and mental health of Lebanese T2DM patients are negatively affected during the COVID-19 pandemic.

First, we analyzed the QoL of Lebanese T2DM patients. We found that the ADDQoL AWI score was -2.2 (possible range -9 to +3 ) showing a negative impact of diabetes on QoL in the current sample of T2DM patients. Likewise, several studies have shown, using different QoL and health status scales, that COVID-19 has had a negative impact on QoL in Australian [25], Croatian [9], and Danish [15] T2DM patients. In the Australian study, QoL was mainly affected for leisure activities, feelings about the future, and emotional well-being [25]. In the Arab world, an Egyptian study performed on a sample of $200 \mathrm{~T} 2 \mathrm{DM}$ patients [11], showed that respectively $64 \%$ and $62 \%$ of patients with T2DM reported poor physical and mental health components on the SF-36 measure of health status. In the current study, gender was not associated with the ADDQoL AWI score while age was significantly correlated with a better score. The relation between gender and QoL is conflicting. Two previous studies performed in Egypt [11] and Iran [12], found that women had poorer physical QoL compared to men while in a third
Table 4 ADDQoL and DTSQs scores during COVID-19 versus pre-COVID-19 pandemic

\begin{tabular}{llll}
\hline & Current study & $\begin{array}{l}\text { Atallah et al. study } \\
{[17]}\end{array}$ & $p$ value \\
\hline ADDQoL score & & & \\
AWI & $-2.7 \pm 2.3$ & $-3.1 \pm 1.9$ & 0.001 \\
Overview I item & $+0.4 \pm 1.2$ & $+1.1 \pm 1.1$ & $<0.001$ \\
Overview II item & $-1.4 \pm 1.0$ & $-1.7 \pm 0.9$ & $<0.001$ \\
DTSQs score & $27.4 \pm 8.8$ & $27.6 \pm 6.9$ & $=0.61$ \\
Perceived frequency of hypoglycemia & $2.7 \pm 1.1$ & $2.0 \pm 1.8$ & $<0.001$ \\
Perceived frequency of hyperglycemia & $3.0 \pm 2.0$ & $2.8 \pm 1.8$ & 0.084 \\
\hline
\end{tabular}

Data are expressed as mean $\pm \mathrm{SD}$

$A W I$ average weighted impact 
study [9] male gender was a significant predictor of worse QoL. Likewise, it has been shown that younger Australian T2DM patients reported lower QoL compared to older ones [25]. Conversely, in Denmark [15] and Iran [12], the oldest age group, compared to the youngest one, had a significant decrease in QoL. Cultural differences may explain these differences between countries; it is possible that in our population older T2DM patients are more prone to manage their QoL. We found that dwelling location outside the city of Beirut has a negative impact on QoL, similarly to what was observed in a Croatian study where living in rural regions was a significant predictor of poorer QoL [9]. This could be explained by the fact that living in urban areas allow a better access to good health management. Also, we found that the absence of physical activity during the COVID-19 pandemic was associated with a worse ADDQoL score. This was also found in 2 other studies from Bangladesh [26] and Netherland [27] where participants who became less active during the pandemic experienced more stress and psychological distress. Moreover, patients having at least one comorbidity (dyslipidemia, diabetic nephropathy, retinopathy, and/or neuropathy) and current smokers had lower ADDQoL score. Similarly, in several reports $[9,11,26,28]$, the presence of diabetic complications and current smoking were associated with lower score in physical domain part. Finally, the current study showed that a shorter duration of diabetes, a better diabetes control (measured by the last HbA1C) and the use of OAD are associated with better QoL scores. This finding can be explained by the fact that OAD are usually used at an earlier stage of the disease at a time when diabetic complications are less frequent.

Then, we assessed treatment satisfaction using the DTSQs. Our results showed that our participants experienced an overall good treatment satisfaction with a median score of 30 over 36 , and our median score of perceived hypoglycemia and hyperglycemia was 3 for a range between 1 and 6. This highlights the fact that our population has an adequate degree of satisfaction, regardless their treatment strategy. The discrepancy between the QoL and treatment satisfaction could be explained by the availability of a good Lebanese healthcare network, which was surprisingly maintained despite the prevailing circumstances in a country facing one of the hardest socio-economic crises. Treatment satisfaction has only been studied in the Saudi RIMODIS study [29] during the COVID-19 pandemic. Similarly to our results, a relatively good degree of treatment satisfaction was found also among Saudi T2DM patients with a median score of 28 versus 30 in the current study).

We also evaluated mental health issues in our population. The K10 score obtained was of 26 over 36 confirming a notable impact of the COVID-19 pandemic on mental health. In a study from Saudi Arabia [16], the average psychological assessment score K10 of T2DM patients during the COVID-19 pandemic was 9.8. This huge difference with our results can be explained by the additional impact of the severe Lebanese economic and social crisis [30] that has severely burdens the Lebanese mental health. In the United Kingdom (UK) [28] and Denmark [10], the reduction in enjoyment of normal daily activities during the COVID-19 pandemic has also led to an increase in psychological distress. We also found that the K10 score was significantly higher in women compared to men. Similarly, other studies $[11,15,28]$ showed an increase in psychological distress in women comparing to men. At the opposite, in the Croatian study [9] men were more affected in the psychological and the spiritual domain. In addition, we found that the absence of income was associated with the worst $\mathrm{K} 10$ score ( score $=30$ ) indicating severe mental disorder in these patients. Worldwide job loss and/or a wage's reduction were common since the beginning of the economic downturn leading to food insecurity, homelessness, psychological distress, and anxiety [31]. We also demonstrated that patients living outside an urban area, as in the Bekaa valley, North, and South Lebanon had higher K10 scores suggesting that living outside the city may increase the mental distress probably due to the absence of leisure activities adapted to the pandemic in these regions. Moreover, the current study proved that lifestyle factors such as exercise and smoking have an impact on mental health. T2DM patients not practicing regular physical activity were more prone to mental disorders in comparison to those with regular physical exercise. This finding was also seen in studies from Iran and India $[13,32]$ where the decrease in healthy lifestyle behaviors, mainly exercise, during the COVID-19 pandemic has led to an increase in psychological distress. This confirms the importance of regular physical exercise to reduce emotional and psychological stress [33]. Current smokers have also higher K10 score confirming the fact that mental disorders are more associated with addiction behaviors such as smoking [34]. Finally, patients with diabetic complications, on OAD treatment, with a worse diabetes control or with a longer duration of the disease had higher K10 scores. Three studies $[10,11,15]$ have shown that patients with diabetic complications were more likely to have mental distress and feelings of social isolation during the COVID-19 pandemic. In addition, Madsen et al. [15] and Ghosh et al. [35] found that since the beginning of the pandemic, patients with high HbAlc showed an increased in social loneliness, psychological and mental distress scores compared to those with low HbA1c. This can be explained by the fact that uncontrolled diabetes increases the risk of diabetes-related complications, and therefore impact negatively mental health. Similarly to our results, in Singapore [36], patients on both OAD and insulin had more mental disorders in comparison to other treatment modalities (OAD only, insulin only). 
Finally, we conducted a comparison of the current results with those of Atallah et al. study [17] performed in 2017 on a sample of 694 Lebanese and Jordanian's T2DM patients. In that study, the authors analyzed the QoL and treatment satisfaction using the same Arabic version of the questionnaires. The unprecedented political, economic, sanitary and social collapse [30] that Lebanon had to face these last 2 years, added to the tragic massive explosion of August 4th 2020 in the Beirut port has certainly caused extensive physical and mental damage to the overall Lebanese population [37]. This has probably worsened the COVID-19 pandemic's impact on the QoL of the Lebanese population, and explain the lower score obtained in the ADDQoL overview I general QoL in our study compared to the Atallah et al. one. However, there was no significant difference in treatment satisfaction between our results and those of Atallah et al. [17] using the DTSQs questionnaire. This result highlights the persistence of high medical qualified standards in our country despite the severe crisis the country is currently passing through. It is also important to mention, that mean score of perceived hypoglycemia was superior in the current study in comparison to those of Atallah et al. [17]. This could be explained by the impact of COVID-19 on patient's food consumption or diet changes [5]. On the contrary, in the Saudi Arabia study [16], the incidence of hypoglycemia or hyperglycemia did not differ significantly between the pre- and post-lockdown phase for COVID-19 pandemic. In addition, the observed decrease in physical activity and the increase in smoking habits in the current study, can be explained by the national lockdown imposed by the government leading to the closure of gyms, wellbeing places, and increase level of stress among our study population.

\section{Limitations}

In terms of limitations, the present study had an important one that is common with most of the survey studies. Collected data, as already mentioned, was based on self-reported questionnaires. Therefore, there is no way to confirm how accurate the collected information is. Moreover, a control group was not included in this study for the K10 questionnaire. Considering that ADDQoL and DTSQ are only used for diabetic populations, Atallah et al. was chosen as a control group. This might explain some differences obtained between the two studies. However, due to similar ethnicity between the two populations, a comparison can be made. In addition, the study did not capture the economic burden associated with the disease's comorbidities. Finally, it is difficult to compare our results to those of the literature considering the use of a multitude of QoL questionnaires.

\section{Conclusion}

In conclusion, this study showed, for the first time in Lebanon, an altered QoL of the Lebanese patients with T2DM during the COVID-19 pandemic. T2DM has a negative impact on multiple dimensions of QoL, treatment satisfaction and mental health. Younger women patients living outside the city of Beirut, with poor diabetic control or with diabetic complications were more prone to an altered QoL. Moreover, those infected with COVID-19 also reported worse QoL, treatment satisfaction and mental health. These findings highlight the need to improve QoL of T2DM patients during the severe sanitary and societal crisis that Lebanon is facing. Developing strategies to provide adequate physical and mental support to this subgroup of our population is mandatory.

\section{Access to questionnaires}

For access to the ADDQoL and DTSQ, please visit https:// www.healthpsychologyresearch.com

Acknowledgements We thank Professor Clare Bradley and Health Psychology Research for providing us the Lebanese version of ADDQoL and the Arabic version of DTSQs. We also wish to extend our special thanks to Professor Clare Bradley for her very useful comments on the manuscript.

Author contributions All authors contributed to the writing and revision of the manuscript. EN, MHG and MB were responsible for the final version of the manuscript. All authors read and approved the final manuscript.

Funding No funding was received to assist with the preparation of this manuscript.

Data availability The data that support the findings of this study are available from the corresponding author upon reasonable request.

Code availability Not applicable.

\section{Declarations}

Conflict of interest The authors declare that they have no conflicts of interest.

Ethics approval This study was approved by the Ethics committee of Saint-Joseph University (Tfem 2022/11) and was performed in accordance with the ethical standards as laid down in the 1964 Declaration of Helsinki.

Informed consent Informed consent was obtained from all individuals included in the study. 


\section{References}

1. Cucinotta D, Vanelli M (2020) WHO declares COVID-19 a pandemic. Acta Bio-Med Atenei Parm 91(1):157-160

2. Rothan HA, Byrareddy SN (2020) The epidemiology and pathogenesis of coronavirus disease (COVID-19) outbreak. J Autoimmun 109:102433

3. Bai Y, Yao L, Wei T, Tian F, Jin D-Y, Chen L et al (2020) Presumed asymptomatic carrier transmission of COVID-19. JAMA 323(14):1406-1407

4. Bloomgarden ZT (2020) Diabetes and COVID-19. J Diabetes 12(4):347-348

5. Rose KJ, Scibilia R (2021) The COVID19 pandemic-perspectives from people living with diabetes. Diabetes Res Clin Pract 173:108343

6. Trikkalinou A, Papazafiropoulou AK, Melidonis A (2017) Type 2 diabetes and quality of life. World J Diabetes 8(4):120-129

7. Measuring the quality of life in diabetic patients: a scoping review (internet). https://www.hindawi.com/journals/jdr/2020/ 5419298/. Accessed 20 Aug 2021.

8. Bradley $\mathrm{C}$, Eschwège $\mathrm{E}$, de Pablos-Velasco $\mathrm{P}$, Parhofer KG, Simon D, Vandenberghe $\mathrm{H}$ et al (2018) Predictors of quality of life and other patient-reported outcomes in the PANORAMA multinational study of people with type 2 diabetes. Diabetes Care 41(2):267-276

9. Gvozdanović Z, Farčić N, Šimić H, Buljanović V, Gvozdanović L, Katalinić $S$ et al (2021) The impact of education, COVID19 and risk factors on the quality of life in patients with type 2 diabetes. Int J Environ Res Public Health 18(5):2332

10. Joensen LE, Madsen KP, Holm L, Nielsen KA, Rod MH, Petersen AA et al (2020) Diabetes and COVID-19: psychosocial consequences of the COVID-19 pandemic in people with diabetes in Denmark-what characterizes people with high levels of COVID-19-related worries? Diabet Med J Br Diabet Assoc 37(7):1146-1154

11. Abdelghani M, Hamed MG, Said A, Fouad E (2021) Evaluation of perceived fears of COVID-19 virus infection and its relationship to health-related quality of life among patients with diabetes mellitus in Egypt during pandemic: a developing country single-center study. Diabetol Int 12:1-9

12. Arab-Zozani M, Hashemi F, Safari H, Yousefi M, Ameri H (2020) Health-related quality of life and its associated factors in COVID-19 patients. Osong Public Health Res Perspect 11(5):296-302

13. Alimehr M, Malayen S, Vafa FS, Tahmasebi MJ, Nikbina M, Doostifar K (2021) The impact of coronavirus disease (COVID-19) on quality of life in diabetic patients. Clin Diabetol 10(3):237-242

14. Hasan SS, Clavarino AM, Mamun AA, Kairuz T (2016) Anxiety symptoms and the risk of diabetes mellitus in Australian women: evidence from 21-year follow-up. Public Health 130:21-28

15. Madsen KP, Willaing I, Rod NH, Varga TV, Joensen LE (2021) Psychosocial health in people with diabetes during the first three months of the COVID-19 pandemic in Denmark. J Diabetes Complications 35(4):107858

16. Alshareef R, Al Zahrani A, Alzahrani A, Ghandoura L (2020) Impact of the COVID-19 lockdown on diabetes patients in Jeddah. Saudi Arabia Diabetes Metab Syndr 14(5):1583-1587

17. Atallah P, Abu-Hijleh O, El-Zaheri M, Andari E, Haddad F, Hajar $\mathrm{H}$ et al (2020) Diabetes management, quality of life and treatment satisfaction in adult population in Jordan and Lebanon, observations from the SIMPLIFY study. J Diabetes Mellit 10(2):720-726

18. Bradley C, Todd C, Gorton T, Symonds E, Martin A, Plowright $\mathrm{R}$ (1999) The development of an individualized questionnaire measure of perceived impact of diabetes on quality of life: the ADDQoL. Qual Life Res Int J Qual Life Asp Treat Care Rehabil 8(1-2):79-91

19. Wee H-L, Tan C-E, Goh S-Y, Li S-C (2006) Usefulness of the Audit of Diabetes-Dependent Quality-of-Life (ADDQoL) questionnaire in patients with diabetes in a multi-ethnic Asian country. Pharmacoeconomics 24(7):673-682

20. Bradley C, Lewis KS (1990) Measures of psychological wellbeing and treatment satisfaction developed from the responses of people with tablet-treated diabetes. Diabet Med J Br Diabet Assoc 7(5):445-451

21. Bradley C (ed) Diabetes Treatment Satisfaction Questionnaire: (DTSQ). In: Handbook of psychology and diabetes. Routledge, New York (1994)

22. Saisho Y (2018) Use of diabetes treatment satisfaction questionnaire in diabetes care: importance of patient-reported outcomes. Int J Environ Res Public Health 15(5):947

23. Bradley C, Gamsu DS (1994) Guidelines for encouraging psychological well-being: report of a Working Group of the World Health Organization Regional Office for Europe and International Diabetes Federation European Region St Vincent Declaration Action Programme for Diabetes. Diabet Med J Br Diabet Assoc 11(5):510-516

24. Anderson TM, Sunderland M, Andrews G, Titov N, Dear BF, Sachdev PS (2013) The 10-item Kessler psychological distress scale (K10) as a screening instrument in older individuals. Am J Geriatr Psychiatry Off J Am Assoc Geriatr Psychiatry 21(7):596-606

25. Sacre JW, Holmes-Truscott E, Salim A, Anstey KJ, Drummond GR, Huxley RR et al (2021) Impact of the COVID-19 pandemic and lockdown restrictions on psychosocial and behavioural outcomes among Australian adults with type 2 diabetes: findings from the PREDICT cohort study. Diabet Med J Br Diabet Assoc. 38(9):e14611

26. Sujan MSH, Tasnim R, Islam MS, Ferdous MZ, Apu MAR, Musfique MM et al (2021) COVID-19-specific diabetes worries amongst diabetic patients: the role of social support and other co-variates. Prim Care Diabetes S1751-9918(21):00113-00123

27. Regeer H, Nieuwenhuijse EA, Vos RC, Jong JCK, van Empelen P, de Koning EJP et al (2021) Psychological factors associated with changes in physical activity in Dutch people with type 2 diabetes under societal lockdown: a cross-sectional study. Endocrinol Diabetes Metab 4(3):e00249

28. Niedzwiedz CL, Green MJ, Benzeval M, Campbell D, Craig P, Demou E et al (2021) Mental health and health behaviours before and during the initial phase of the COVID-19 lockdown: longitudinal analyses of the UK Household Longitudinal Study. J Epidemiol Commun Health 75(3):224-231

29. AlSlail FY, Akil YA (2021) Treatment patterns, effectiveness, and satisfaction among patients with type 2 diabetes treated with insulin in Saudi Arabia: results of the RIMODIS study. Diabetes Ther 12(7):1965-1978

30. Lebanon sinking into one of the most severe global crises episodes, amidst deliberate inaction (internet). World Bank. https:// www.worldbank.org/en/news/press-release/2021/05/01/leban on-sinking-into-one-of-the-most-severe-global-crises-episodes. Accessed 20 Aug 2021

31. Forde R, Arente L, Ausili D, De Backer K, Due-Christensen M, Epps A et al (2021) The impact of the COVID-19 pandemic on people with diabetes and diabetes services: A pan-European survey of diabetes specialist nurses undertaken by the Foundation of European Nurses in Diabetes survey consortium. Diabet Med J Br Diabet Assoc. 38(5):e14498

32. Varshney M, Parel JT, Raizada N, Sarin SK (2020) Initial psychological impact of COVID-19 and its correlates in Indian 
Community: an online (FEEL-COVID) survey. PLoS ONE 15(5): 0233874

33. Sharma A, Madaan V, Petty FD (2006) Exercise for mental health. Prim Care Companion J Clin Psychiatry 8(2):106

34. Boksa P (2017) Smoking, psychiatric illness and the brain. J Psychiatry Neurosci JPN 42(3):147-149

35. Ghosh A, Arora B, Gupta R, Anoop S, Misra A (2020) Effects of nationwide lockdown during COVID-19 epidemic on lifestyle and other medical issues of patients with type 2 diabetes in north India. Diabetes Metab Syndr 14(5):917-920

36. Yeoh E, Tan SG, Lee YS, Tan HH, Low YY, Lim SC et al (2021) Impact of COVID-19 and partial lockdown on access to care, self-management and psychological well-being among people with diabetes: a cross-sectional study. Int J Clin Pract 75(8):e14319

37. Devi S (2020) Lebanon faces humanitarian emergency after blast. Lancet Lond Engl 396(10249):456

Publisher's Note Springer Nature remains neutral with regard to jurisdictional claims in published maps and institutional affiliations. 